\title{
5 Research Square

\section{METTL13 Mediates the Translation of Snail in Head and Neck Squamous Cell Carcinoma}

\section{Xiaochen Wang}

Sun Yat-sen University First Affiliated Hospital

\section{Kang Li}

Sun Yat-sen University First Affiliated Hospital

Yuehan Wan

Sun Yat-Sen University

\section{Maosheng Cheng}

Sun Yat-sen University First Affiliated Hospital

\section{Gan Xiong}

Sun Yat-Sen University

\section{Ganping Wang}

Sun Yat-sen University First Affiliated Hospital

\section{Shuang Chen}

Sun Yat-sen University First Affiliated Hospital

\section{Zhi Chen}

Sun Yat-sen University First Affiliated Hospital

Jianwen Chen

Sun Yat-sen University First Affiliated Hospital

\section{Xiuyun Xu}

Sun Yat-Sen University

Cheng Wang

Sun Yat-Sen University

\section{Liang Peng}

Chinese PLA General Hospital

Demeng Chen ( $\nabla$ chendm29@mail.sysu.edu.cn)

Sun Yat-sen University First Affiliated Hospital

\section{Research}

Keywords: HNSCC, METTL13, EMT, Snail

Posted Date: November 5th, 2020 
DOI: https://doi.org/10.21203/rs.3.rs-101161/v1

License: (c) (1) This work is licensed under a Creative Commons Attribution 4.0 International License. Read Full License 


\section{Abstract}

Background: Accumulating evidence supports that Methyltransferase like 13 (METTL13), which is implicated in protein binding and synthesis, act as oncogenic molecule. This study aimed to reveal the potential mechanism of METTL13 in head and neck squamous cell carcinoma (HNSCC) via mediating enhancing translation efficiency of Snail.

Methods: The expression of METTL13, Snail and ki67 were detected in patient HNSCC and paired paracarcinoma tissues. Knockdown and ectopic expression experiments were used in HNSCC cells to investigate their effects on tumor cell migration, proliferation and apoptosis. RNA-sequencing, polysome profiling and SUnSET assays were developed to obtain the further mechanism of the interaction among METTL13, Snail and translation.

Results: METTL13 was significantly upregulated in HNSCC at both mRNA and protein level. Increased METTL13 was negatively associated with clinical prognosis. And METTL13 markedly affected HNSCC cellular phenotypes in vivo and vitro. Further mechanism study revealed that METTL13 could regulate EMT signaling pathway by mediating enhancing translation efficiency of Snail, the key transcription factor in EMT, hence regulating the progression of EMT.

Conclusion: Altogether, our study had revealed the clinical significance of METTL13 in HNSCC, and providing a potential therapeutic strategy.

\section{Background}

Human head and neck squamous cell carcinoma (HNSCC) is one of the most commonly diagnosed malignancies worldwide with high mortality and morbidity, making it a growing health burden $[1,2]$. And this highly malignant disease is characterized by poor prognosis due to its high local recurrence and lymph node metastasis [3]. As for the metastasis of malignant tumor, epithelial-mesenchymal transition (EMT) was considered to be a crucial part $[4,5]$. It was activated in tumor progression $[6,7]$ and affected cancer stem cell cellular behaviors [8,9]. During EMT, a small subset of cancer cells acquire motility with decreased adhesive ability and obtain invasive properties. Several EMT-induced transcription factors, such as Slug, Twist, Snail, have been considered to be involved in the progress [10]. Specifically, Snail, a major transcription factor governing EMT, equip cancer cells with malignant phenotype like tumor recurrence and metastasis. Furthermore, although many of the signaling pathways could impinge on the transcriptional regulation of the Snail gene [11], post-transcriptional mechanisms affecting Snail are also emerging as crucial regulators of its activity [12].

Especially, aberrant regulation of mRNA translation to protein often account for the progression of cancer [13]. For instance, the subunits of the eukaryotic translation initiation factor $4 \mathrm{~F}$ (elF4F) complex and eukaryotic elongation factor 2 (eEF2), were frequently found to be dysregulated in multiple cancer types $[14,15]$. Increased activities of translation regulators enable the cancer cells to rapidly produce selective proteins in response to cancer development. For example, eukaryotic translation elongation factor 1 
alpha 2 (eEF1A2) can mediate the expression of EMT factor matrix metallopeptidase 9 (MMP-9) in pancreatic cancer [16]. In addition, chemical modification of translation regulators by phosphorylation, acetylation, methylation and ubiquitination can result in changes of translation efficiency. For instance, phosphorylation of elF2a leads to the gene-specific translation, which further support cell survival in human cancers $[17,18]$.

Recently, studies reported that human methyltransferase like 13 (METTL13) can catalyze dimethylation of eukaryotic elongation factor $1 \mathrm{~A}(\mathrm{eEF} 1 \mathrm{~A})$ lysine 55 (eEF1AK55me2) to promote the protein synthesis in Ras-driven cancers $[19,20]$. METTL13 was characterized with oncogenicity, as it was involved in various diseases, such as gastric cancer [21], lung cancer [22,23] and breast cancer [24]. METTL13 also functions as a potential inhibitor of apoptosis, for it was found that miR-16 promoted apoptosis of tumor cells by silencing protein synthesis through posttranscriptional regulation [25]. Collectively, these findings suggest that METTL13 could be a crucial gene of tumorigenesis and development.

In the present study, we examined the expression of METTL13 in paired HNSCC and para-carcinoma samples, and found METTL13 was significantly increased in HNSCC. Further study confirmed it was related to overall survival. And METTL13 knockdown significantly inhibits the proliferation and induced apoptosis in vitro. Conversely, the overexpression of METTL13 can promote malignant phenotypes of tumor. By RNA-seq, we figured out that METTL13 regulated the EMT pathway. In terms of mechanism, we managed to demonstrate that METTL13 could enhance translation efficiency of Snail, the key factor in EMT, hence regulating the progression of EMT. Therefore, our data revealed the function and underlying mechanism of METTL13 in HNSCC, providing both a prognostic marker and drug candidate for this malignancy.

\section{Materials And Methods}

\subsection{Cell culture and patient samples.}

Cal27, Cal33 and SCC1 cell lines were obtained from Type Culture Collection of the Chinese Academy of Sciences in Shanghai, China. And all the cells were cultured in Dulbecco's Modified Eagle's Medium (DMEM, Gibco, 11885-076) and supplemented with 10\% fetal bovine serum (FBS, Gibco, 10270-106) and $1 \%$ penicillin-streptomycin (Gibco, 15140122 ) at a $37^{\circ} \mathrm{C}$ incubator containing $5 \% \mathrm{CO}_{2}$.

Both of the HNSCC tissues and adjacent tissues were collected from HNSCC patients who underwent surgery at the Hospital of Stomatology, Sun Yat-sen University. This study received the informed written consent from all patients before the experiment.

\subsection{Cell transfection}

METTL13 gene expression was inhibited by two sequences of shRNAs, as showed in Table 1. The synthesized and purified METTL13 gene and control fragment were imported into a lentivirus vector. And recombinant lentivirus was produced from 293T cells by LipofectamineTM 2000 transfection reagent 
(Thermo Fisher Scientific, 11668019), and transfected into HNSCC cell lines by polybrene (8 $\mu \mathrm{g} / \mathrm{ml})$. And pFLAG-CMV2-METTL13 plasmid was used for overexpression of METTL13.

\subsection{Total RNA extraction and real-time fluorescent quantitative PCR.}

The total RNA of cells was obtained according to the instructions provided by the TRIZOL reagent (Invitrogen, 15596026). After that, $1 \mu \mathrm{g}$ mRNA was utilized to reverse to cDNA by the standard procedure of the PrimeScript RT master mix kit (TaKaRa, RR036A). Next, TB Green ${ }^{\text {TM }}$ Premix Ex Taq II kit (TaKaRa, RR820B) was used for real-time PCR according to the instructions and operated under the Bio-Rad CXF96 real-time system (Bio-Rad, USA). $\beta$-actin was functioned as internal control to calculate the relative quantity. The primers used for realtime PCR were listed in Table 2.

\subsection{Total protein extraction and western blotting analysis.}

The total protein of HNSCC cell lines was harvested by with ice-cold radioimmu-noprecipitation assay (RIPA) lysis solution containing 1\% PMSF protease inhibitor. And Pierce ${ }^{\text {TM }}$ BCA Protein Assay Kit (Thermo Fisher Scientific, 23227) had been used to define protein concentration in cell lysates. Before the western blot assay, the protein was dissolved in loading buffer and denatured at high temperature for ten minutes. And the cell protein component was isolated by $12 \%$ SDS polyacrylamide gel electrophoresis and transferred to the polyvinylidene fluoride membrane (PVDF, Millipore, Germany). After 5\% skimmed milk blocking for one hour, the primary antibody was added to the PVDF membrane and placed overnight at $4{ }^{\circ} \mathrm{C}$. Enhanced chemiluminescence (ECL) reagents were used to detect the protein antibody complexes. And the following primary antibodies were used: Snail (Cell Signaling Technology, 3879T), Twist (Cell Signaling Technology, 46702S), Slug (Cell Signaling Technology, 9585T), MMP7 (Abcam, ab216631100ul), MMP9 (Abcam, ab74277-100ul), E-cadherin (Affinity, AF0131-100), N-cadherin (Affinity, AF403950ul), Vimentin (Abcam, ab24525-50ul), METTL13(GeneTex, GTX120626-S). GADPH (1:1,000, Abcam) was served as internal control.

\subsection{Colony formation assay}

Two-hundred Cal27 or Cal33 cells were added into 6-well plates and were cultivated for about 2 weeks. Cells were fixed for 30 minutes after 14 days with 4 percent paraformaldehyde and stained with 0.1 percent violet crystal. Each group had been repeated in triplicate.

\subsection{Cell proliferation assay.}

Cell proliferation ability was assessed via CCK8 assay (Dojindo, CCK8-500). According to the standard procedure, $100 \mu \mathrm{l}$ cell suspension containing $2 \times 10^{3}$ cells was seeded into each well of the 96 -well plate and cultured in a cell incubator. At equal time intervals (four times every 24 hours), $10 \mu$ reaction agent was added to each well and cultured for 1 hour at $37^{\circ} \mathrm{C}$, and then taken out for absorbance value detection at $450 \mathrm{~nm}$ (Infinite 200 PRO). 
As for the wound healing experiment, cells were transplanted and grown until they developed a monolayer of 90 percent fusion. Cells were then removed with the tip of an aseptic straw and treated in FBS-free medium according to the instructions. The scratched visual field was randomly selected under the microscope, and the migration distance of the cells to the scratched area was measured.

Transwell system was utilized for migration analysis. The cells were cultured in pure DMEM in the cell culture insert (Merck millipore, MCHT06H48) with a final concentration of $1 \times 10^{4} / \mathrm{ml}$. And DMEM containing $10 \%$ FBS coated in the bottle of 24 -well plate was served as a chemical inducer. The cells were fixed with $4 \%$ paraformaldehyde at $4{ }^{\circ} \mathrm{C}$ for 30 minutes after 18 hours of incubation at $37{ }^{\circ} \mathrm{C}$ and $5 \% \mathrm{CO}_{2}$. Cells were stained with crystal violet after rinsing with PBS and incubated for 45 minutes at room temperature. The cells on the cell culture insert's upper side were erased with a cotton swab, and washed thoroughly with PBS. and washed thoroughly with PBS. The average number of cells was calculated according to the random visual field. Each sample were counted for three chambers.

\subsection{Flow cytometric analysis of Annexin $V$ apoptosis and cell cycle assay.}

Cell apoptosis rate was detected using flow cytometry as per the Annexin $\mathrm{V}$ apoptosis detection kit (Dojindo, AD10) instructions. HNSCC cell lines were transplanted into 6-well plates $\left(1 \times 10^{5} / \mathrm{ml}\right.$ per well) for apoptosis analysis and cultivated at $37^{\circ} \mathrm{C}$ for $48 \mathrm{~h}$ in complete medium. Cells were then harvested and stained with the kit after the treatment with non-EDTA trypsin. HNSCC cells used for tracking the cell cycle were added into 6-well plates $\left(5 \times 10^{5} / \mathrm{ml}\right.$ per well) and grown overnight in complete medium at $37^{\circ} \mathrm{C}$. Cells were collected after digestion with non-EDTA trypsin, and fixed with $75 \%$ ethanol overnight. And cells were stained with Kit (Multiscience, 70-CCS012) for 15 min the next day. Both of the two experiments were performed on a flow cytometer with BD FACSDiva Software 6.0 (BD Biosciences, New York, NY, USA). All the experiments were repeated at least three times.

\subsection{Flow cytometric analysis of Aldehyde dehydrogenase activity assay.}

Aldehyde dehydrogenase activity assay was conducted by the ALDEFLUOR fluorescence reagent system (Stem Cell Technology, 01700). In short, we labeled each sample with a test tube and a control tube. The cells were lysed and collected into the test tube and activated ALDEFLUOR reagent was added to each cell suspension. Half of the cell fluid in the test tube was added to the control tube, and $5 \mu \mathrm{l}$ diethylaminobenzaldehyde (DEAB) were immediately added to the control tube. Incubated at $37^{\circ} \mathrm{C}$ for 45 minutes and centrifuged. Then the supernatant was removed and re-suspended in the determination buffer. Finally, the analysis was performed on the flow cytometry.

\subsection{IHC Staining and H\&E Staining}

IHC and H\&E staining procedure had been described previously [26]. All the primary antibodies including METTL13 (GeneTex, GTX120626-S), Snail (Cell Signaling Technology, 3879T) and Ki67 (Affinity, AF0198) was used at dilution in 1:100. IHC staining intensity was scored as 0 (negative), 1 (weak), 2 (medium), or 
3 (strong). And IHC score was defined by multiplying the percentage of positive cells $(P)$ by the intensity (I). Formula: Q = P x l; Maximum = 300 .

\subsection{RNA-sequencing}

Cal27 and Cal33 cells transfected with shCtrl, shRNA-1 and shRNA-2 were sent for RNA-sequencing. Briefly, the total RNA was Total RNA was extracted using TRIZOL as mentioned previously. RNA quality and purity were checked by Nano-300.

Following the Illumina protocol, the construction of cDNA library was achieved from RNA samples for Illumina paired-end (PE) sequencing. The library preparations were subsequently sequenced on a platform of Illumina HiSeq 2000 (Illumina, San Diego, CA) and paired-end reads (2100 bp) were produced at Novogene Bioinformatics Technology Co., Ltd (Beijing, China).

\subsection{SUnSET assay.}

As for translation analysis, the cells were incubated with puromycin (Beyotime, ST551-50 mg) of $1 \mu \mathrm{M}$ final concentration at $37^{\circ} \mathrm{C}$ for $30 \mathrm{~min}$. After that the cells were collected and lysed in RIPA buffer containing with $1 \mathrm{mM}$ PMSF and protease inhibitor. The whole cell lysate of 5-10 $\mu \mathrm{g}$ was detected by western blotting with anti-puromycin primary antibody (Sigma-Aldrich, MABE343).

\subsection{Polysome Profiling and fractionation}

Polysome profiling assay was conducted as described previously [27]. In short, Cal27 and Cal33 cells with control or METTL13-decreased were cultured in $15 \mathrm{~cm}$ dishes (about $10 \times 10^{6}$ cells in each dish). Then cells were loaded with actinomycin at $100 \mu \mathrm{g} / \mathrm{ml}$, then incubated for 2 minutes at $37^{\circ} \mathrm{C}$. Then cells were collected and cell extract was loaded on a sucrose gradient of $10-50 \%$ and prepared with a BioComp gradient station. The gradient was rotated at $36000 \mathrm{rpm}$ and $4{ }^{\circ} \mathrm{C}$ for 2 hours in the TH-641 rotor (Sorvall). The gradient $(260 \mathrm{~nm})$ was analyzed and the components were collected by the BioComp gradient station.

\subsection{Sphere Formation Assays.}

According to the method we used before [28], the stem cell culture medium containing $3 \times 10^{3} \mathrm{cells} / \mathrm{ml}$ will be put into the 6-well ultralow attachment plates. Incubate for $7-10$ days in a $37^{\circ} \mathrm{C}$ incubator containing $5 \% \mathrm{CO}_{2}$. The stem cell culture medium was replaced every two days. After spheres were formed, they were digested into single sphere by $0.25 \%$ Trypsin-EDTA. Photographs and counts were taken under a microscope.

\subsection{Animal studies}

As for the in vivo subcutaneous transplanted model studies, four-week-old BALB/C female nude mice obtained from Center of Experimental Animal of Sun Yat-sen University were divided into three groups ( $\mathrm{n}$ $=6$ per group) at random. HNSCC cell lines that transfected with METTL13-deficient or control plasmid 
were subcutaneously injected into mice to explore the effect in vivo. After four weeks, all the mice were sacrificed and we resected xenograft tumors intactly. The volume of transplanted tumor volume was determined as follows: length $\times$ width ${ }^{2} / 2$. All the experimental studies were performed in the Sun Yat-sen University Laboratory Animal Centre. All procedures of animal experiments were approved by Sun Yat-sen University Animal Care and Use Committee.

\subsection{Statistical analysis}

The experiments had been replicated at least three times for in vitro research, each experiment was performed in duplicate or triplicate. The sample size necessary for vivo studies to discern difference in statistical significance is at least 6 animals in each group.

Data was analyzed by unpaired Student t-test to assess statistical significance. Data was shown as mean \pm SD. All the mathematical analysis of the data was done by GraphPad Prism 8.0.

\section{Results}

\section{METTL13 expression was upregulated in HNSCC and negatively correlated with clinical survival.}

To investigate the expression pattern of METTL13 in HNSCC, we firstly analyzed its mRNA expression level by qPCR in 67 HNSCC and paired para-carcinoma tissues obtained from Hospital of Stomatology of Sun Yat-sen University. Notably, significant upregulation of METTL13 was observed $(p<0.001$, Fig. 1A). Consistently, TCGA analysis data also showed that METTL13 was upregulated in the HNSCC samples (Fig. 1B). Next, we examined the METTL13 expression at protein level by immunohistochemistry (IHC). Higher expression of METTL13 was observed in human HNSCC samples than paired normal tissues (Fig. 1C). To further explore the potential prognostic value in HNSCC, a total of 44 patients were segmented into METTL13 low and high expression groups based on the GPCR results. The data showed METTL13 significantly negatively correlated with overall survival of HNSCC patients $(p<0.05$, Fig. 1D). Collectively, we showed that the expression of METTL13 was upregulated in HNSCC, and it might play a key role in HNSCC development and progression.

\section{Knockdown of METTL13 inhibited HNSCC cell malignant phenotypes in vitro.}

Aberrant cellular activities often occur in cancer cells, such as continuous proliferative, evading growth inhibition, resisting cell apoptosis and active invasion and metastasis [29]. To explore the functional role of METTL13 in HNSCC cells, we first selected eight HNSCC cell lines and compared their METTL13 expression by the Western blotting assay (Fig. 2A). The results revealed high METTL13 protein level in two HNSCC cancer cell lines (Cal27 and Cal33) and low METTL13 protein level in SCC1 cell line (Fig. 2A). Hence, we chose Cal27, Cal33 and SCC1 cell lines for further functional study. We then generated stable METTL13 knockdown Cal27 and Cal33 cell lines using shRNA targeting METTL13 and confirmed the successful knockdown of METTL13 gene at mRNA and protein levels (Fig. 2B, C). Next, we performed clone formation assay and CCK-8 assay to explore the effect of METTL13 gene knockdown on the 
proliferation in HNSCC cell lines. The results showed that the depletion of METTL13 significantly not only inhibited the clone formation ability, but also inhibited cell growth rate in these two cell lines (Fig. 2D, E). Next, we utilized a flow cytometer to study the role of METTL13 in cell apoptosis. Our results demonstrated a higher apoptosis rate after ablation of METTL13 in HNSCC cells (Fig. 2F). Subsequently, wound healing assay results revealed that silencing of METTL13 significantly suppressed cell migration and prolonged the repair time (Fig. 2G). Transwell cell migration assay further proved knockdown of METTL13 attenuated the invasion ability of HNSCC cells (Fig. 2H). Taken together, these results demonstrated that METTL13 is critical for proliferation, apoptosis and invasion of HNSCC cell lines.

\section{Mettl13 Downregulation Negatively Correlates With Hnscc Csc-like Properties}

We also investigated whether METTL13 played a role in HNSCC cancer stem cells (CSCs)-like properties. First, we determined HNSCC cellular self-renewal capacity by conducting the sphere-forming assay. Downregulation of METTL13 greatly reduced the size and number of tumor spheres when compared with those formed by control cells in both Cal27 and Cal33 cell lines (Fig. 3A, B), indicating that their ability to self-renew was impaired. We then further assessed the CSC-like properties of METTL13 using assay to detect aldehyde dehydrogenase (ALDH) activity, which was regarded as a reliable marker for CSCs [30]. As showed in Fig. 3C-D, ALDH activity was significantly reduced in cells treated with sh-METTL13. Therefore, METTL13 played an essential part in maintaining HNSCC cells self-renewal ability.

\section{Overexpression of METTL13 could promote HNSCC tumorigenicity.}

In further explore the function of METTL13, a stable METTL13 overexpression SCC1 cell line was generated. And the up-regulation efficiency was verified by western blotting and qPCR assays (Fig. 4A, B). The CCK-8 assay revealed overexpression of METTL13 remarkably increased the capacity of HNSCC cells to proliferate (Fig. 4C). Consistently, lower rate of apoptotic cells was captured in METTL13 ${ }_{\text {over }}$ group by the flow cytometry (Fig. 4D). Next, we found that extra acquisition of METTL13 could accelerated cell migration of SCC1 in the transwell assay (Fig. 4E). Furthermore, METTL13 ${ }_{\text {over }}$ group tended to form larger size of tumor spheres after having cultured for seven days (Fig. 4F). On the other hand, overexpression of METTL13 endowed SCC1 cells with higher ALDH activities (Fig. 4G). To sum up, these data show that METTL13 plays a potential oncogenic role by increasing or inducing the malignant proliferation, migration and CSC-like properties in HNSCC cells.

\section{METTL13 regulated EMT signaling pathway by enhancing translation efficiency of Snail to facilitate HNSCC progress}

We have demonstrated that METTL13 expressly promoted HNSCC proliferation and metastasis, but the specific mechanism was unclear. Previous studies have reported that METTL13-mediated methylation of eukaryotic elongation factor $1 \mathrm{~A}(\mathrm{eEF} 1 \mathrm{~A})$ promote oncogenesis via increased translation elongation and 
protein synthesis in cancer cells [19]. And indeed, our polysome profile assay results revealed that decrease of METTL13 suppressed total protein translation in both Cal27 and Cal33 cell lines (Fig. 5A). In addition, the surface sensing of translation (SUnSET) monitoring method confirmed protein synthesis was inhibited after METTL13 depletion (Fig. 5B).

Previous study has indicated that METTL13 is involved in RAS-driven cancer development, we wonder whether depletion of METTL13 in HNSCC is also related to RAS signaling pathway [19]. Therefore, RNA sequencing was performed using the METTL13 control and knockdown groups in both Cal27 and Cal33 cell lines. Interestingly, results from Gene Set Enrichment Analysis (GSEA) indicated that EMT signaling pathway was significantly negatively correlated with the downregulation of METTL13 in HNSCC cell lines (Fig. 5C), suggesting that downregulation of protein synthesis might prone to EMT-related factors. To examine whether this is the case, a series of key proteins associated with EMT pathway were examined by western blotting (Fig. 5D, data not shown). Notably, as a key transcription factor in EMT, Snail was significantly downregulated in METTL13-deficient cells (Fig. 5D). Therefore, we hypothesized that METTL13 could regulate EMT signaling pathway by regulating the translation activity of Snail mRNA. To test that, we performed polysome profile assay using sucrose gradient centrifugation to separate the ribosome and polysome segments. Based on these results, we used qPCR to detect the distribution of METTL13 affected mRNAs in the polysome segment. And the results showed Snail mRNA was significantly down-regulated in the METTL13 deficient groups in the polysome levels (Fig. 5E), indicating that the METTL13 is required for translation of Snail, which consistent with our previous conjecture. Altogether our results support that Snail is involved in METTL13-regulated EMT in HNSCC, and METTL13 regulated EMT signaling pathway by enhancing translation efficiency of Snail.

\section{Decreased METTL13 expression weakened malignant phenotypes in vivo.}

Xenograft studies were then conducted to investigate whether METTL13 could recapitulate the in vitro malignant phenotypes in vivo. Control and two METTL13 shRNA Cal33 cell lines were subcutaneous injected into nude mice. Mice were sacrificed after four weeks. In accordance with our in vitro results, deficiency of METTL13 in Cal33 cell dramatically suppressed tumor growth when compared with the control in vivo (Fig. 6A). And the tumor volume and tumor weight results further confirmed this effect (Fig. 6B, C). In order to get a more profound understanding of the function of METTL13 in vivo, we obtain the tumor sections. Next, hematoxylin and eosin (H\&E) staining and METTL13 IHC results verified that METTL13 expression was decreased in sh-METTL13 tumor cells compared to the control (Fig. 6D, E). The staining of Ki67, a proliferation marker, showed alleviated cell growth after METTL13 gene knockdown, which was consistent with in vitro results (Fig. 6E). Consistently, IHC staining of nude mice showed the expression of Snail protein was reduced by the silencing of METTL13 (Fig. 6E).

Collectively, these data demonstrated that METTL13 is required for tumorigenic ability of HNSCC cells in vivo.

\section{Discussion}


Translation is one of the most energy consumption processes inside the cell and dysregulation of translation mechanisms is a common cause of neoplastic diseases [31,32]. Because in cancer cells where mRNA translation is usually highly active, it is necessary to increase the rate of protein synthesis [14]. And increasing evidences revealed translation elongation factor modification could cause promotion or inhibition of protein synthesis [33,34]. In our study, we demonstrated that METTL13, a regulator of translation elongation, showed higher expression levels in HNSCC tumors than adjacent normal tissues and might serve as a prognostic marker for overall survival in HNSCC patients.

METTL13 has been reported to be involved in cell apoptosis, stem cell biology and various cell signal and metabolic pathways [35]. However, whether it participates in tumorigenesis activity in HNSCC remains largely elusive. Here, we documented a detailed oncogenic role of METTL13 in HNSCC cell lines. We found that METTL13 is necessary and required for proliferation and EMT of HNSCC cancer cells both in vitro and in vivo. The translation level of METTL13-depleted HNSCC cells underwent significantly decrease compared with control cells. Intriguingly, GESA analysis of the transcriptome showed that genes down-regulated in the METTL13-depleted HNSCC are related to EMT process. Previously, METTL13 has been shown to promote cell proliferation and metastasis by elevating the levels of TCF3 and ZEB in hepatocellular carcinoma [36]. In addition, METTL13 can participate in development of Ras-dependent epithelial tumors of the pancreas and lung [19]. In HNSCC, we found depletion of METTL13 profoundly reduced the protein level of Snail, one of the key transcription factors of EMT. More specifically, we demonstrated that METTL13 could enhance the translation efficiency of Snail mRNA to promote EMT and HNSCC progression. It is highly possible that the inhibition of the metastatic ability of cancer cells after deletion of METTL3 may be mitigated by Snail's overexpression. Previous studies revealed that EMT could be utilized by the tumor cells to perform metastatic cascades [7], and scientific groups began to work on the its underlying mechanisms in tumor progression. In one study, EMT signaling pathway was blocked through modulation of the key protein translation in breast cancer [37]. Thereby, our study provided a more profound understanding of EMT in HNSCC.

\section{Conclusion}

In conclusion, our data revealed the tumor promoter function of METTL13 in HNSCC. METTL13 could regulate the progression of EMT by promoting Snail mRNA translation. These results suggested that METTL13 might function as a promising drug target. METTL13 could regulate the translation of downstream proteins by affecting the stability of $80 \mathrm{~s}$ ribosome structure. Although deeper researches are needed to uncover the mechanism of METTL13 carcinogenesis and as a drug target for HNSCC in the future, our present research has built the foundation for better therapeutic strategies.

\section{Abbreviations}

METTL13 methyltransferase like 13

HNSCC head and neck squamous cell carcinoma 
EMT epithelial-mesenchymal transition

elF4F eukaryotic translation initiation factor $4 \mathrm{~F}$

eEF2 eukaryotic elongation factor 2

eEF1A2 eukaryotic translation elongation factor 1 alpha 2

MMP-9 matrix metallopeptidase 9

eEF1AK55me2 eukaryotic elongation factor $1 \mathrm{~A}$ lysine 55

ALDH aldehyde dehydrogenase

CSC cancer stem cell

FITC Fluorescein isothiocyanate

PI Propidium iodide

WT Wild type

GAPDH Glyceraldehyde-3-phosphate dehydrogenase

sh Short hairpin

GSEA Gene-set enrichment analysis

SUnSET surface sensing of translation

E-cad E-cadherin

MMP7 matrix metallopeptidase 7

MMP9 matrix metallopeptidase 9

\section{Declarations}

\section{Ethics approval and consent to participate}

All the animal experimental studies were performed in the Sun Yat-sen University Laboratory Animal Centre. All procedures of animal experiments were approved by Sun Yat-sen University Animal Care and Use Committee. This study received the informed written consent from all patients before the experiment.

\section{Consent for publication}

Not applicable. 
Availability of data and materials

The datasets generated and/or analyzed during the current study are available from the corresponding author on reasonable request.

\section{Competing interests}

The authors indicate no potential competing interests.

\section{Funding}

The Natural Science Foundation of Guangdong Province (no. 1814050001877) and the National Natural Science Foundation of China (no. 8187111008).

\section{Authors' contributions}

DC, LP and CW conceived and designed this project. XW and MC conducted experiments and interpreted results of experiments. KL drafted paper, edited and revised manuscript. YW, GX, GW and SC perform patients' data analysis. ZC, JC and XX prepared figures. All authors read and approved the final manuscript.

\section{Acknowledgements}

The authors would like to acknowledge the helpful suggestions of Ashwini Nikam from Springer Nature Transfer Desk.

\section{Footnote}

Xiaochen Wang, Kang Li and Yuehan Wan are shared co-first authorship.

\section{References}

1. Bray 1TorreLA, Siegel F, Ferlay RL, Lortet-Tieulent J, Jemal J A. Global cancer statistics, 2012. CA Cancer J Clin. 2015;65(2):87-108. 'doi:'10.3322/caac.21262.

2. Braakhuis 2LeemansCR, Brakenhoff BJ RH. The molecular biology of head and neck cancer. NAT REV CANCER. 2011;11(1):9-22. 'doi:'10.1038/nrc2982.

3. Forastiere 3MarurS, Head AA. and Neck Squamous Cell Carcinoma: Update on Epidemiology, Diagnosis, and Treatment. MAYO CLIN PROC. 2016;91(3):386 - 96. 'doi:'10.1016/j.mayocp.2015.12.017.

4. Pastushenko I, Blanpain C. EMT Transition States during Tumor Progression and Metastasis. TRENDS CELL BIOL. 2019;29(3):212 - 26. 'doi:'10.1016/j.tcb.2018.12.001.

5. Kudo-Saito C, Shirako H, Takeuchi T, Kawakami Y. Cancer metastasis is accelerated through immunosuppression during Snail-induced EMT of cancer cells. CANCER CELL. 2009;15(3):195-206. 
'doi:'10.1016/j.ccr.2009.01.023.

6. Lamouille S, Xu J, Derynck R. Molecular mechanisms of epithelial-mesenchymal transition. Nat Rev Mol Cell Biol. 2014;15(3):178 - 96. 'doi:'10.1038/nrm3758.

7. Mittal V. Epithelial Mesenchymal Transition in Tumor Metastasis. Annu Rev Pathol. 2018;13:395412. 'doi:'10.1146/annurev-pathol-020117-043854.

8. Zhou P, Li B, Liu F, Zhang M, Wang Q, Liu Y, et al. The epithelial to mesenchymal transition (EMT) and cancer stem cells: implication for treatment resistance in pancreatic cancer. MOL CANCER. 2017;16(1):52. 'doi:'10.1186/s12943-017-0624-9.

9. Lee SY, Jeong EK, Ju MK, Jeon HM, Kim MY, Kim CH, et al. Induction of metastasis, cancer stem cell phenotype, and oncogenic metabolism in cancer cells by ionizing radiation. MOL CANCER. 2017;16(1):10. 'doi:'10.1186/s12943-016-0577-4.

10. Nieto MA, Huang RY, Jackson RA, Thiery JPEMT. 2016. CELL. 2016;166(1):21-45. 'doi:'10.1016/j.cell.2016.06.028.

11. Thiery JP, Sleeman JP. Complex networks orchestrate epithelial-mesenchymal transitions. Nat Rev Mol Cell Biol. 2006;7(2):131 - 42. 'doi:'10.1038/nrm1835.

12. Sureban SM, May R, Qu D, Weygant N, Chandrakesan P, Ali N, et al. DCLK1 regulates pluripotency and angiogenic factors via microRNA-dependent mechanisms in pancreatic cancer. PLOS ONE. 2013;8(9):e73940. 'doi:'10.1371/journal.pone.0073940.

13. Leibovitch $M$, Topisirovic I. Dysregulation of mRNA translation and energy metabolism in cancer. Adv Biol Regul. 2018;67:30 - 9. 'doi:'10.1016/j.jbior.2017.11.001.

14. Bhat M, Robichaud N, Hulea L, Sonenberg N, Pelletier J, Topisirovic I. Targeting the translation machinery in cancer. NAT REV DRUG DISCOV. 2015;14(4):261 - 78. 'doi:'10.1038/nrd4505.

15. Ng TH, Sham K, Xie CM, Ng S, To KF, Tong J, et al. Eukaryotic elongation factor-2 kinase expression is an independent prognostic factor in colorectal cancer. BMC CANCER. 2019;19(1):649. 'doi:'10.1186/s12885-019-5873-0.

16. Xu C, Hu DM, Zhu Q. eEF1A2 promotes cell migration, invasion and metastasis in pancreatic cancer by upregulating MMP-9 expression through Akt activation. Clin Exp Metastasis. 2013;30(7):933 - 44. 'doi:'10.1007/s10585-013-9593-6.

17. Zhou D, Palam LR, Jiang L, Narasimhan J, Staschke KA, Wek RC. Phosphorylation of elF2 directs ATF5 translational control in response to diverse stress conditions. J BIOL CHEM. 2008;283(11):7064-73. 'doi:'10.1074/jbc.M708530200.

18. Vattem KM, Wek RC. Reinitiation involving upstream ORFs regulates ATF4 mRNA translation in mammalian cells. Proc Natl Acad Sci U S A. 2004;101(31):11269-74. 'doi:'10.1073/pnas.0400541101.

19. Liu S, Hausmann S, Carlson SM, Fuentes ME, Francis JW, Pillai R, et al. METTL13 Methylation of eEF1A Increases Translational Output to Promote Tumorigenesis. CELL. 2019;176(3):491-504. 'doi:'10.1016/j.cell.2018.11.038. 
20. Jakobsson ME, Malecki JM, Halabelian L, Nilges BS, Pinto R, Kudithipudi S, et al. The dual methyltransferase METTL13 targets N terminus and Lys55 of eEF1A and modulates codon-specific translation rates. NAT COMMUN. 2018;9(1):3411. 'doi:'10.1038/s41467-018-05646-y.

21. Wang Q, Chen C, Ding Q, Zhao Y, Wang Z, Chen J, et al. METTL3-mediated m(6)A modification of HDGF mRNA promotes gastric cancer progression and has prognostic significance. GUT. 2020;69(7):1193 - 205. 'doi:'10.1136/gutjnl-2019-319639.

22. Lin S, Choe J, Du P, Triboulet R, Gregory RI. The m(6)A Methyltransferase METTL3 Promotes Translation in Human Cancer Cells. MOL CELL. 2016;62(3):335 - 45. 'doi:'10.1016/j.molcel.2016.03.021.

23. Jin D, Guo J, Wu Y, Du J, Yang L, Wang X, et al. m(6)A mRNA methylation initiated by METTL3 directly promotes YAP translation and increases YAP activity by regulating the MALAT1-miR-1914-3pYAP axis to induce NSCLC drug resistance and metastasis. J HEMATOL ONCOL. 2019;12(1):135. 'doi:'10.1186/s13045-019-0830-6.

24. Sun T, Wu Z, Wang $X$, Wang $Y$, Hu X, Qin W, et al. LNC942 promoting METTL14-mediated m(6)A methylation in breast cancer cell proliferation and progression. ONCOGENE. 2020;39(31):5358-72. 'doi:'10.1038/s41388-020-1338-9.

25. Liang $H$, Fu Z, Jiang $X$, Wang $N$, Wang F, Wang $X$, et al. miR-16 promotes the apoptosis of human cancer cells by targeting FEAT. BMC CANCER. 2015;15:448. 'doi:'10.1186/s12885-015-1458-8.

26. Wang X, Li K, Cheng M, Wang G, Han H, Chen F, et al. Bmi1 Severs as a Potential Tumor-Initiating Cell Marker and Therapeutic Target in Esophageal Squamous Cell Carcinoma. STEM CELLS INT. 2020;2020:8877577. 'doi:'10.1155/2020/8877577.

27. Lin X, Chai G, Wu Y, Li J, Chen F, Liu J, et al. RNA m(6)A methylation regulates the epithelial mesenchymal transition of cancer cells and translation of Snail. NAT COMMUN. 2019;10(1):2065. 'doi:'10.1038/s41467-019-09865-9.

28. Chen D, Wu M, Li Y, Chang I, Yuan Q, Ekimyan-Salvo M, et al. Targeting BMI1(+) Cancer Stem Cells Overcomes Chemoresistance and Inhibits Metastases in Squamous Cell Carcinoma. CELL STEM CELL. 2017;20(5):621 - 34. 'doi:'10.1016/j.stem.2017.02.003.

29. Hanahan D, Weinberg RA. Hallmarks of cancer: the next generation. CELL. 2011;144(5):646 - 74. 'doi:'10.1016/j.cell.2011.02.013.

30. Chen YC, Chen YW, Hsu HS, Tseng LM, Huang PI, Lu KH, et al. Aldehyde dehydrogenase 1 is a putative marker for cancer stem cells in head and neck squamous cancer. Biochem Biophys Res Commun. 2009;385(3):307 - 13. 'doi:'10.1016/j.bbrc.2009.05.048.

31. Pedersen MT, Jensen KB. Cell biology: Unconventional translation in cancer. NATURE. 2017;541(7638):471-2. 'doi:'10.1038/nature21115.

32. Lindqvist LM, Tandoc K, Topisirovic I, Furic L. Cross-talk between protein synthesis, energy metabolism and autophagy in cancer. CURR OPIN GENET DEV. 2018;48:104 - 11. 'doi:'10.1016/j.gde.2017.11.003. 
33. Talavera A, Hendrix J, Versees W, Jurenas D, Van Nerom K, Vandenberk N, et al. Phosphorylation decelerates conformational dynamics in bacterial translation elongation factors. SCI ADV. 2018;4(3):p9714. 'doi:'10.1126/sciadv.aap9714.

34. Hamey JJ, Wilkins MR. Methylation of Elongation Factor 1A: Where, Who, and Why? TRENDS BIOCHEM SCI. 2018;43(3):211 - 23. 'doi:'10.1016/j.tibs.2018.01.004.

35. Takahashi A, Tokita H, Takahashi K, Takeoka T, Murayama K, Tomotsune D, et al. A novel potent tumour promoter aberrantly overexpressed in most human cancers. Sci Rep. 2011;1:15. 'doi:'10.1038/srep00015.

36. Li L, Zheng YL, Jiang C, Fang S, Zeng TT, Zhu YH, et al. HN1L-mediated transcriptional axis AP2gamma/METTL13/TCF3-ZEB1 drives tumor growth and metastasis in hepatocellular carcinoma. CELL DEATH DIFFER. 2019;26(11):2268-83. 'doi:'10.1038/s41418-019-0301-1.

37. Wang WD, Shang Y, Li Y, Chen SZ. Honokiol inhibits breast cancer cell metastasis by blocking EMT through modulation of Snail/Slug protein translation. ACTA PHARMACOL SIN. 2019;40(9):1219-27. 'doi:'10.1038/s41401-019-0240-x.

\section{Tables}

Due to technical limitations, table 1 and 2 is only available as a download in the Supplemental Files section.

\section{Figures}


A

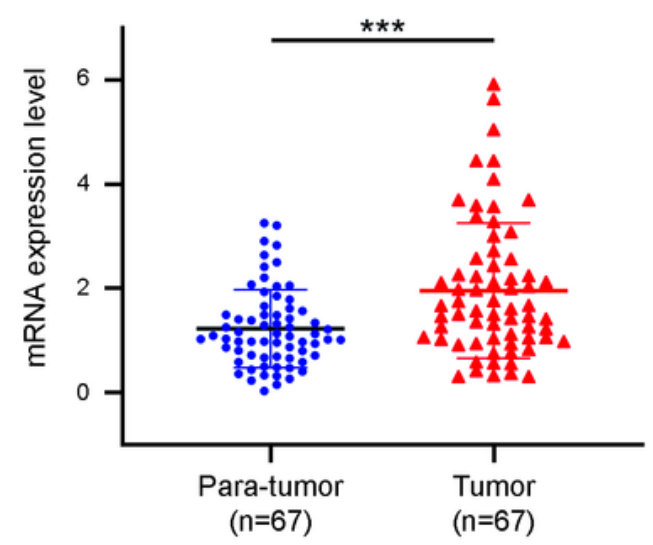

C

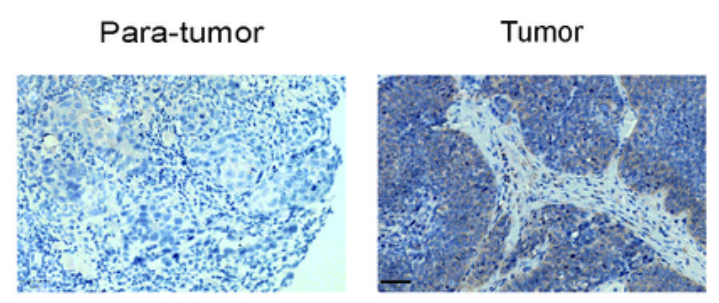

METTL13

D

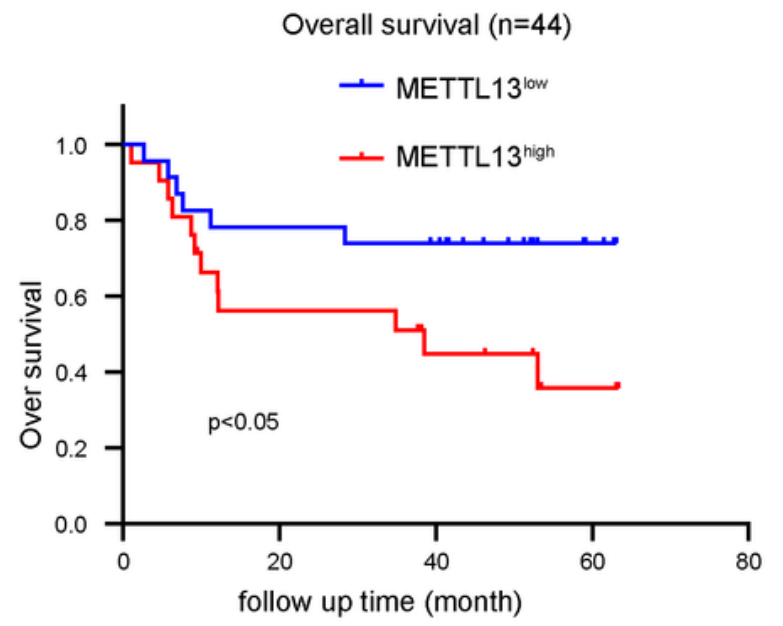

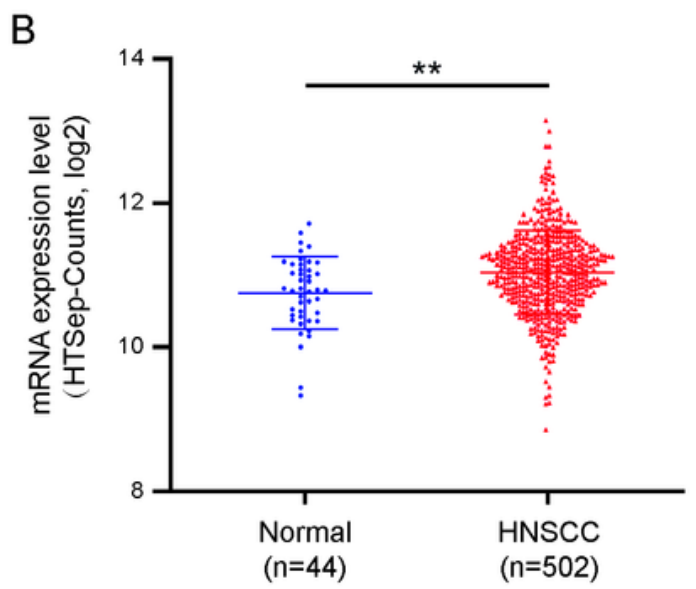

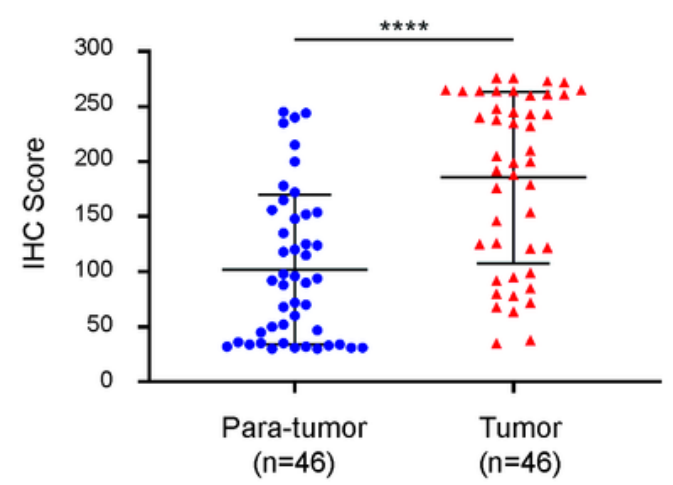

\section{Figure 1}

The expression of METTL13 is increased in HNSCC and its high expression is correlated with poor prognosis. A. Real-time PCR analysis of METTL13 mRNA level in 67 pairs HNSCC tumor tissues and paired adjacent nontumor tissues. The METTL13 mRNA levels are normalized to the $\beta$-actin RNA levels. B. Scatter diagram comparing levels of METTL13 mRNA in normal tissues and HNSCC tissues in published data from TCGA database, ${ }^{* * P}<0.01$ by t-tests. C. IHC staining of METTL13 in HNSCC and 


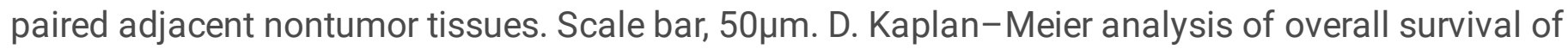
patients $(n=44)$. Patients were divided into METTL13 low group and METTL13 high group according to the Real-time PCR results.
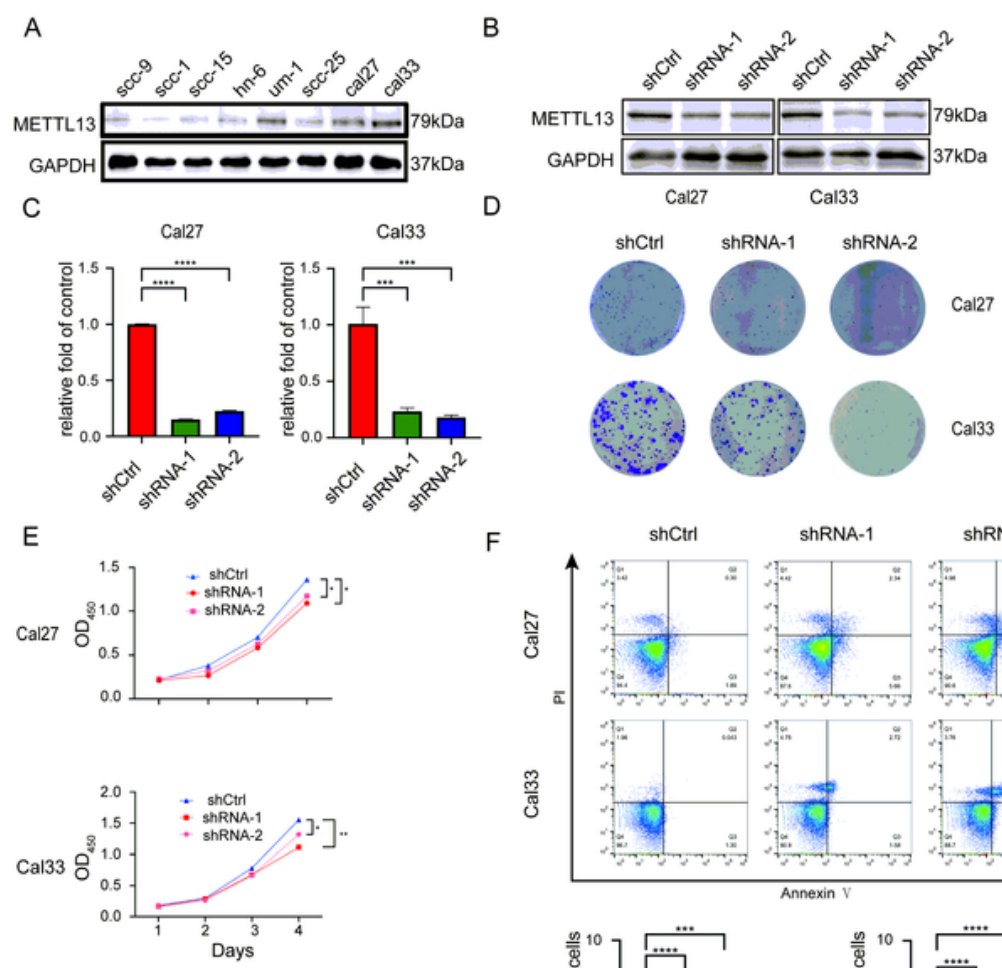

G
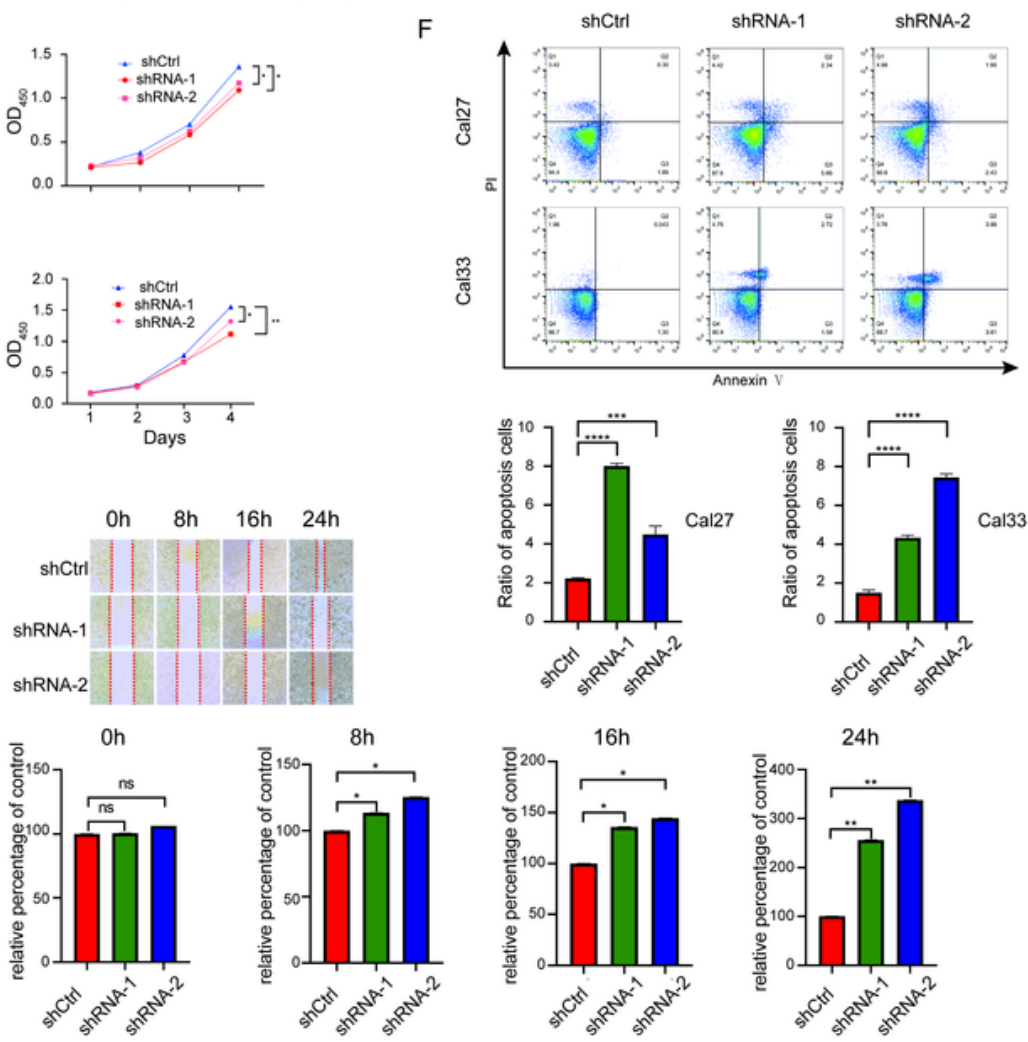

$\mathrm{H}$
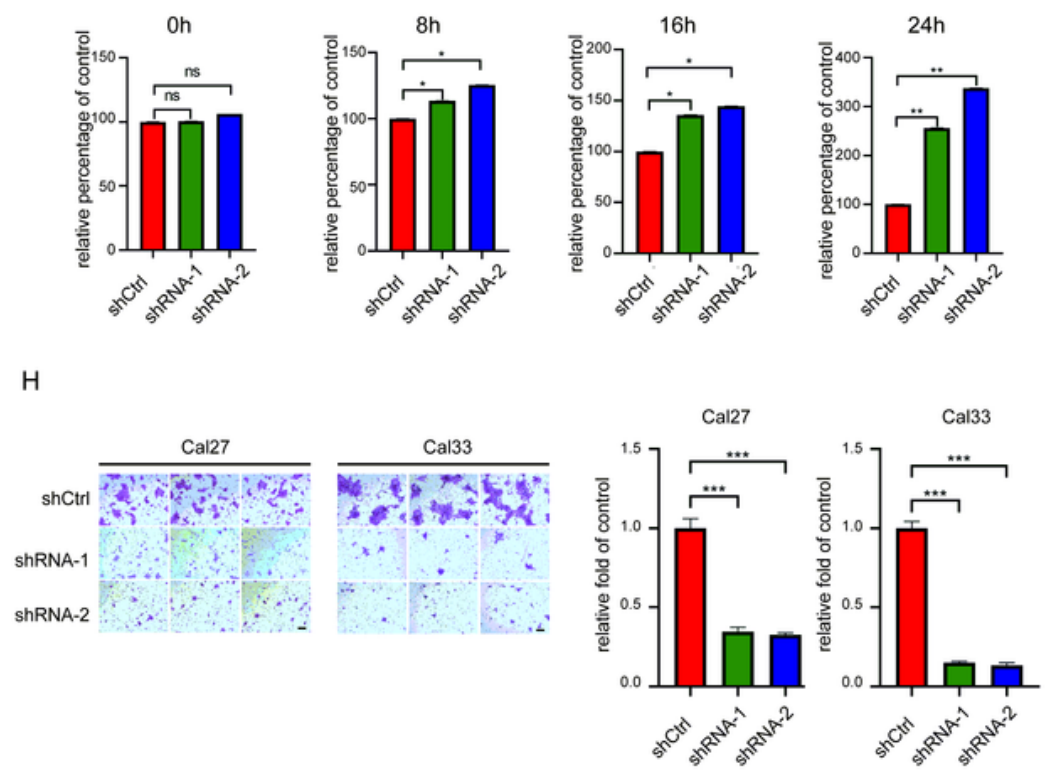

Figure 2

Knockdown of METTL13 attenuates cell proliferation in vitro. A. Western blot analysis of METTL13 expression in different HNSCC cells. GAPDH was served as internal control. B-C. Knockdown efficiency of 
METTL13 in Cal27 and Cal33 cells was confirmed by western blot and real-time PCR analysis. D. The colony formation assay was performed on Cal27 and Cal33 cells transfected with LV-shRNA-1, LV-shRNA2 and LV-shCtrl, colony numbers were measured after crystal violet staining. E. The CCK8 assay examining the effect of METTL13 decrease on the proliferation of Cal27 and Cal33 cells. The experiments were performed in triplicate. F Cell apoptosis rate of Cal27 and Cal33 cells stable silenced METTL13 expression was detected by flow cytometry. Ratio of early and late apoptotic cells was collected and presented in the column chart. G. Knockdown of METTL13 decreased cell migration capacity, as determined by the wound-healing assay. $\mathrm{H}$. Cell migration abilities of Cal27 and Cal33 cells stably transfected with vector or shRNA were evaluated by transwell migration assay. Scale bar, $100 \mu \mathrm{m}$. 

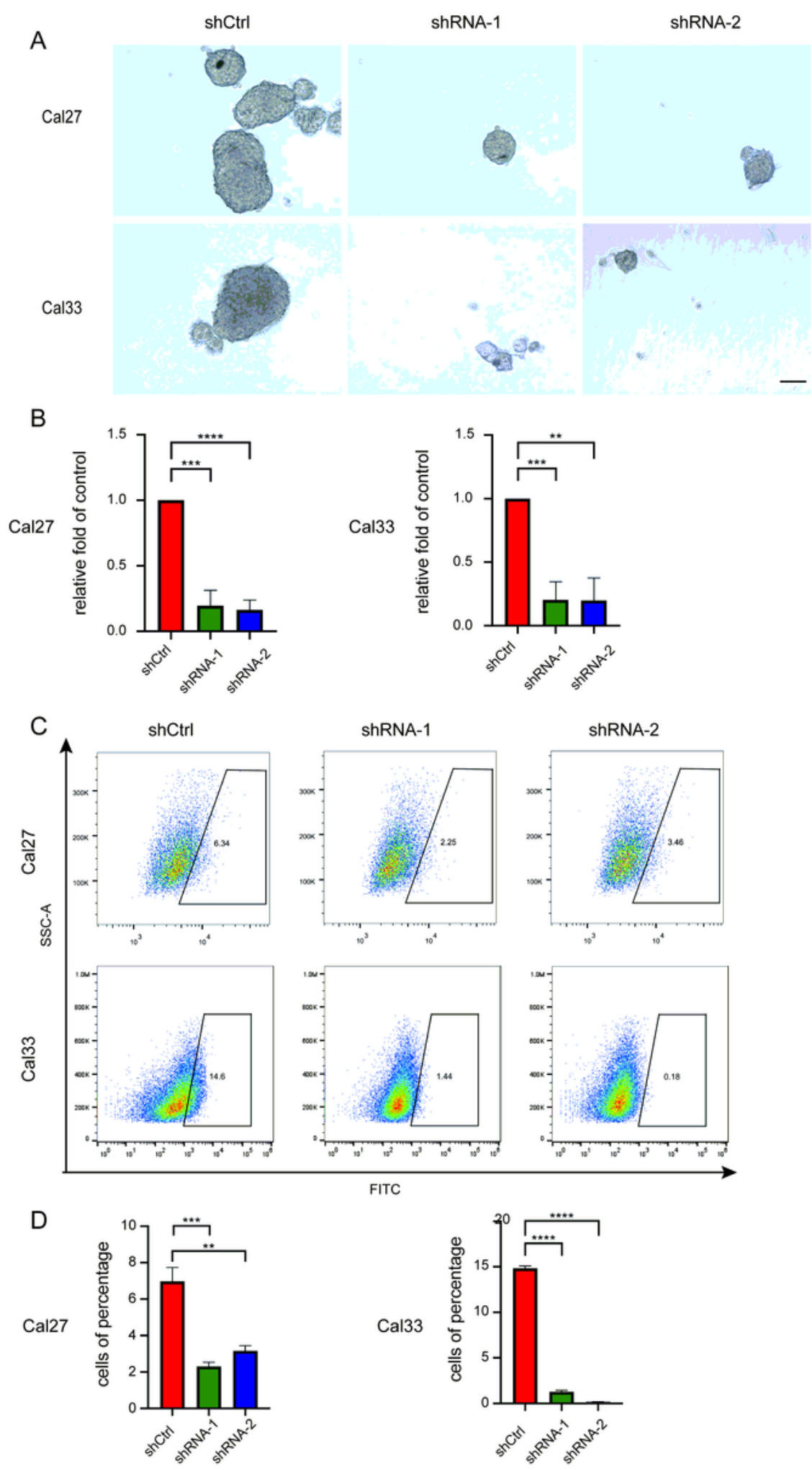

\section{Figure 3}

METTL13 was demonstrated to be associated with HNSCC CSC-like properties. A-B. Representative images and quantification results of sphere-forming assay for Cal27 and Cal33 transfected with LVshRNA-1, LV-shRNA-2 and LV-shCtrl. C. Flow cytometry analysis of ALDH activity of Cal27 and Cal33 cells. D. Column chart of cells with ALDH activity. ${ }^{\star \star P}<0.01$, ${ }^{\star \star \star} P<0.001,{ }^{\star \star \star \star *} \mathrm{P}<0.0001$ by t-tests. 
A

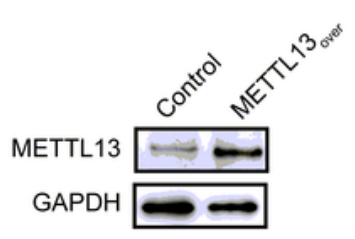

D

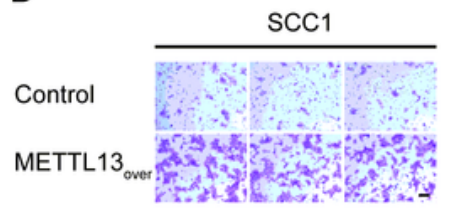

$\mathrm{E}$
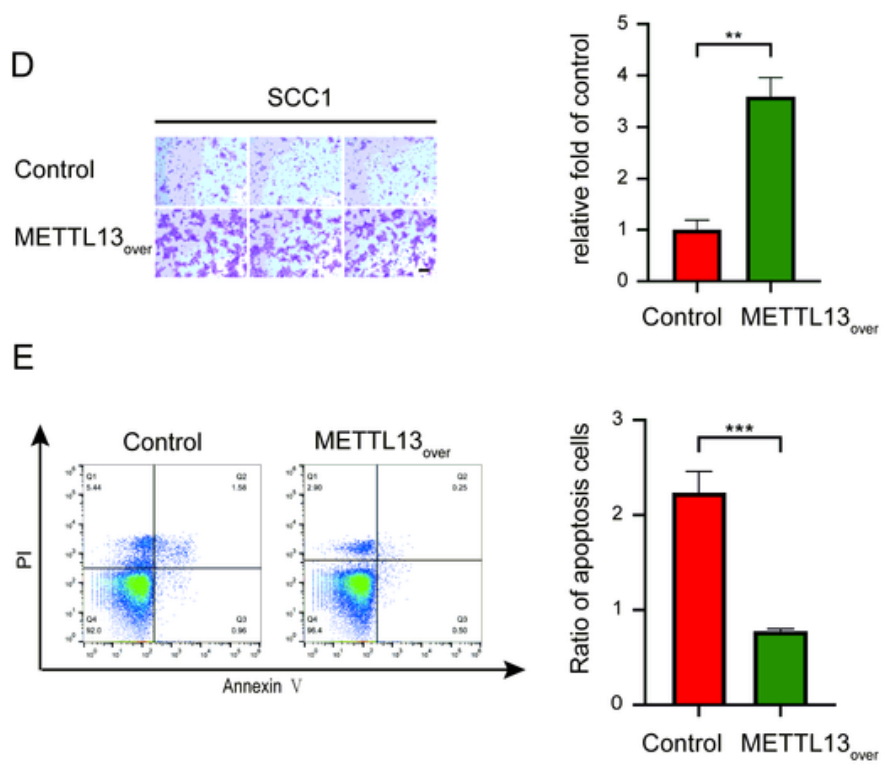
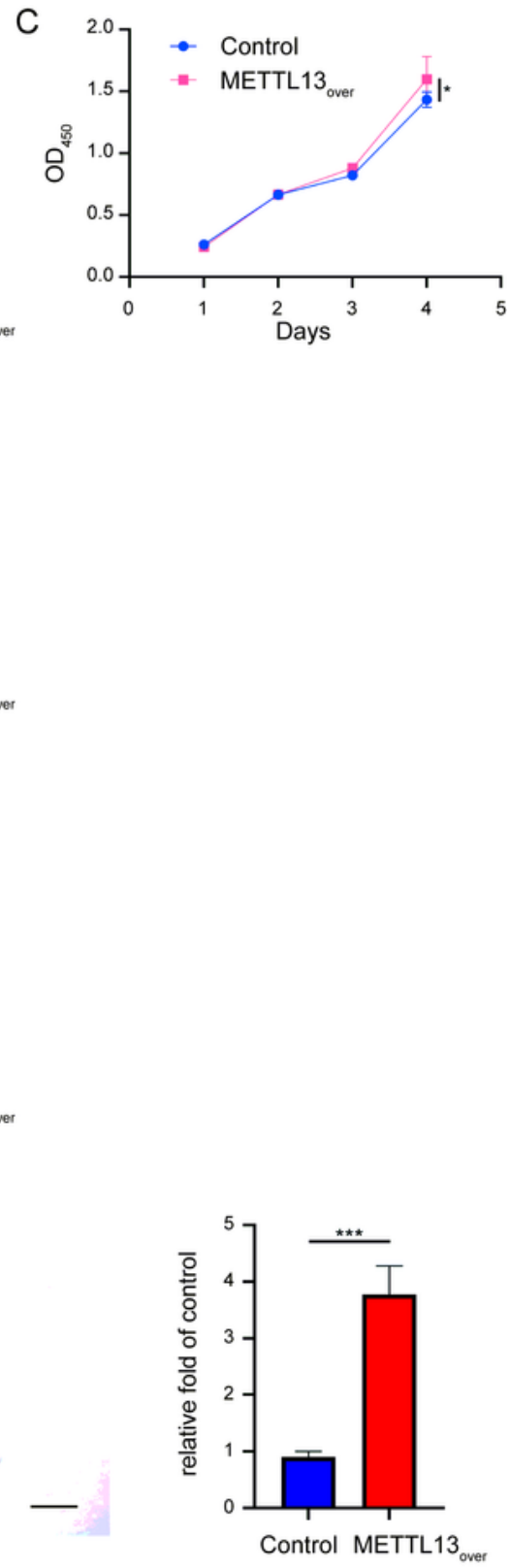

METTL13 $3_{\text {over }}$

G
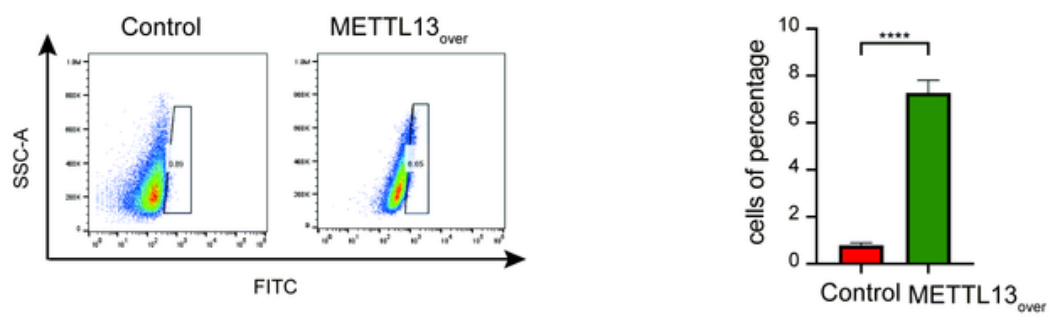

Figure 4

Overexpression of METTL13 promotes HNSCC cell malignant phenotype. A-B. The expression levels of METTL13 in SCC1 METTL13-overexpressing cells or corresponding negative control were confirmed by western blot and real-time PCR analysis. C. The CCK8 assay examining the effect of METTL13 overexpression on the growth of SCC1 cells. D. Cell migration abilities of SCC1 cells with METTL13

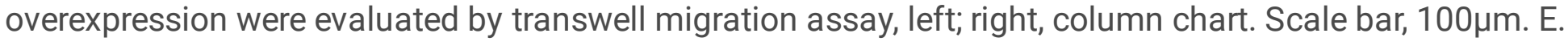


Cell apoptosis rate was examined by flow cytometry using the Annexin $\mathrm{V}$ apoptosis detection kit. Ratio of early and late apoptotic cells was collected and presented in the column chart. F. Representative images and quantification results of sphere-forming assay for SCC1 cell lines. G. ALDH activity was detected by the flow cytometry to examine the effect of METTL13 overexpression on the cellular stemness.

A

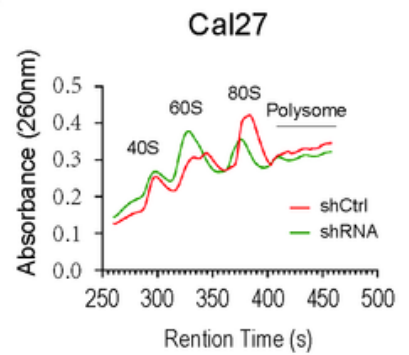

C

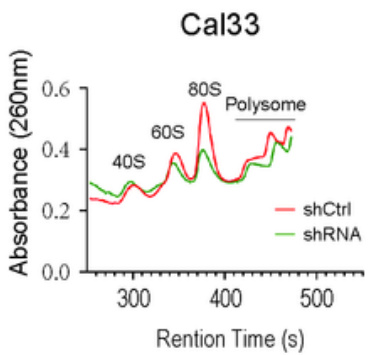

Cal27

$$
\text { Enrichment plot: }
$$

HALLIMARK_EPITHELIAL_MESENCHYMAL_TRANSITION

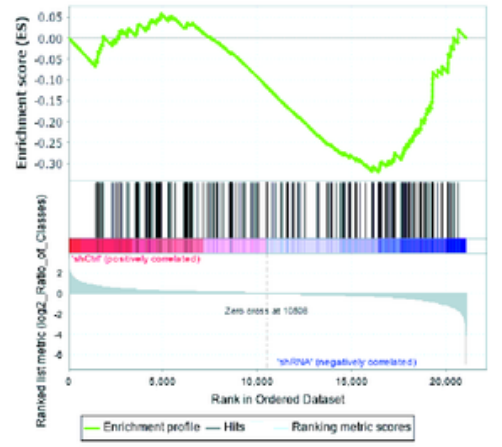

Cal33

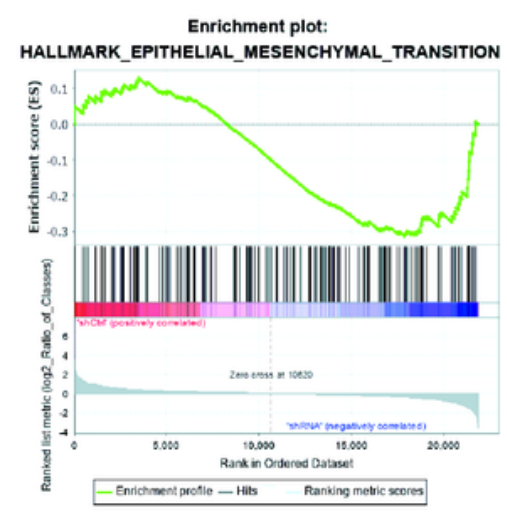

B

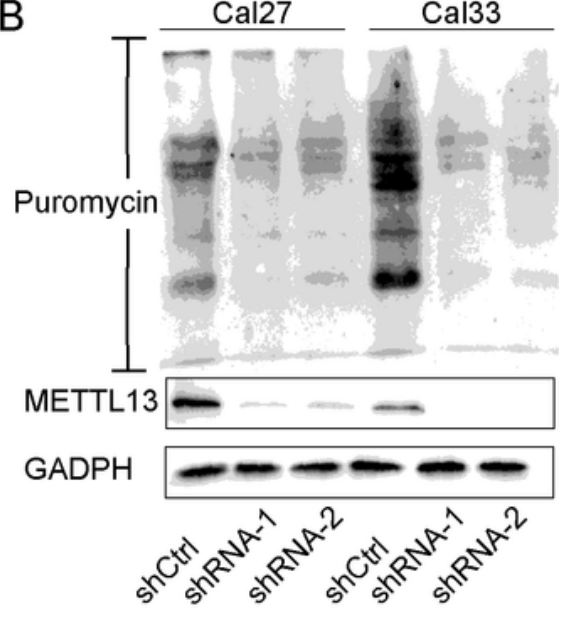

D
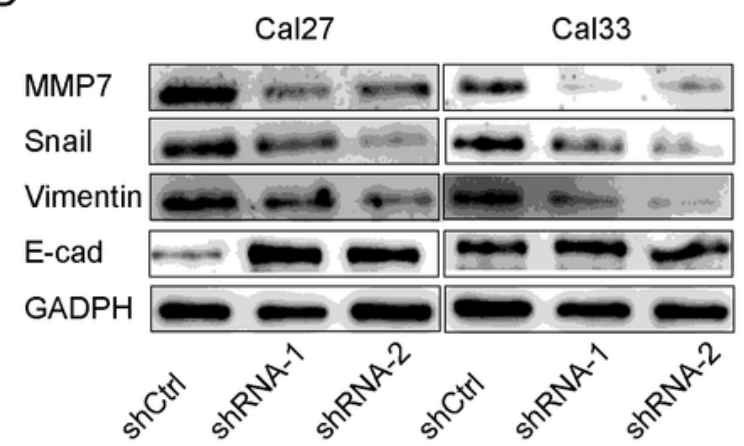

$\mathrm{E}$
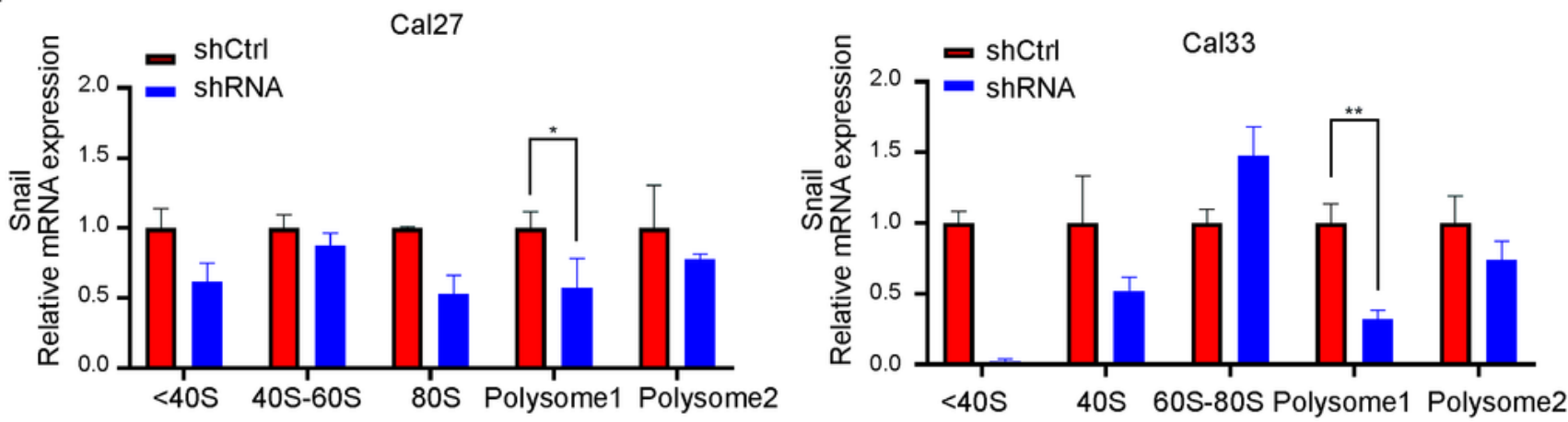

Figure 5 
METTL13 promotes translation of Snail to regulate EMT in HNSCC cancer cells. A. Polysome profiling of wild-type (shCtrl) or Mettl13 knockdown (shRNA) in Cal27 and Cal33 cells were analyzed. B. SUnSET assays were used to confirm METTL13 decrease could suppress translation of HNSCC cells. C.

Representative GSEA curves for significant enriched gene sets related to EMT pathways upon METTL13 silencing compared to shCtrl cells. D. Western blot analysis of EMT relevant molecules in METTL13knockdown cell lines. GAPDH is used as an internal control. E. Analysis of Snail mRNA in non-translation segment (<40S), 40S, 60S, 80S, and polysome for the Mettl13 knockdown cells compared to control cells. $\beta$-actin was functioned as internal control.

A

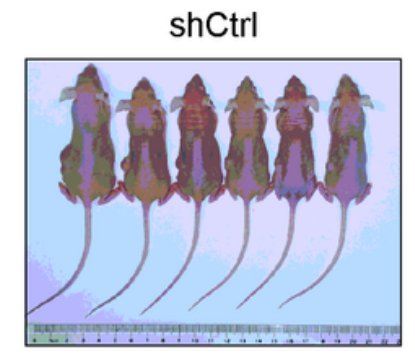
shRNA-1

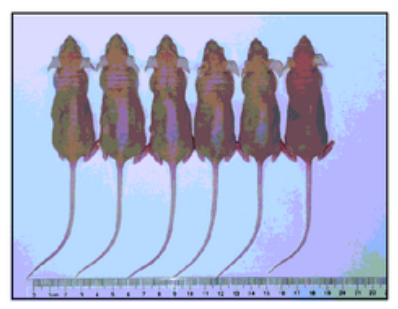

B

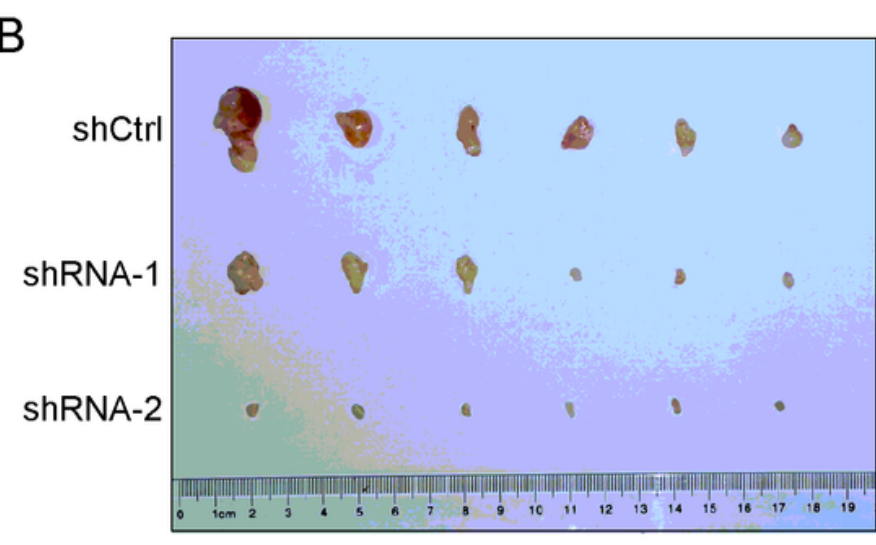

D

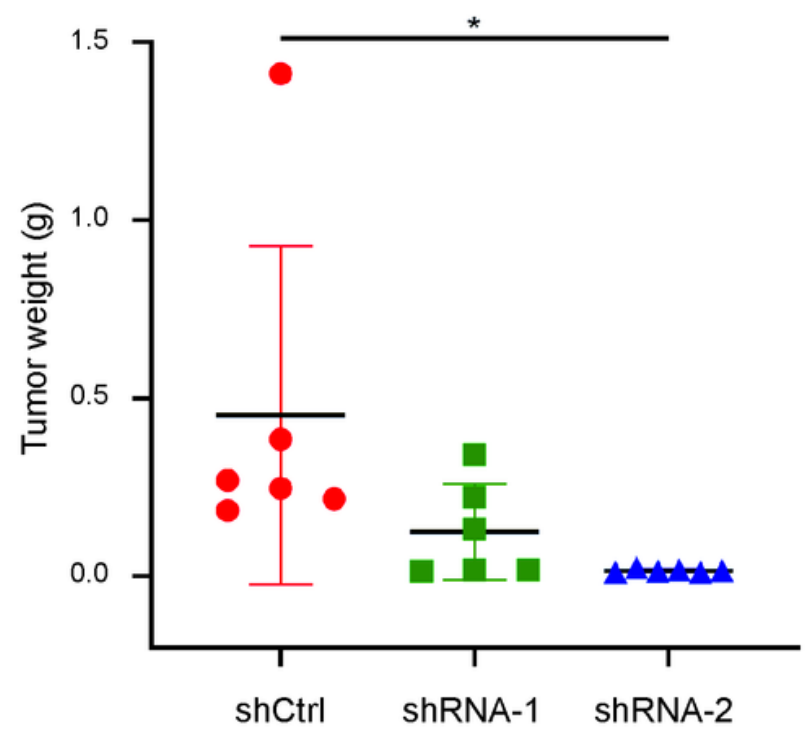

ShRNA-2

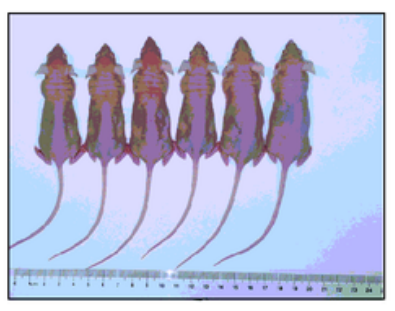

C

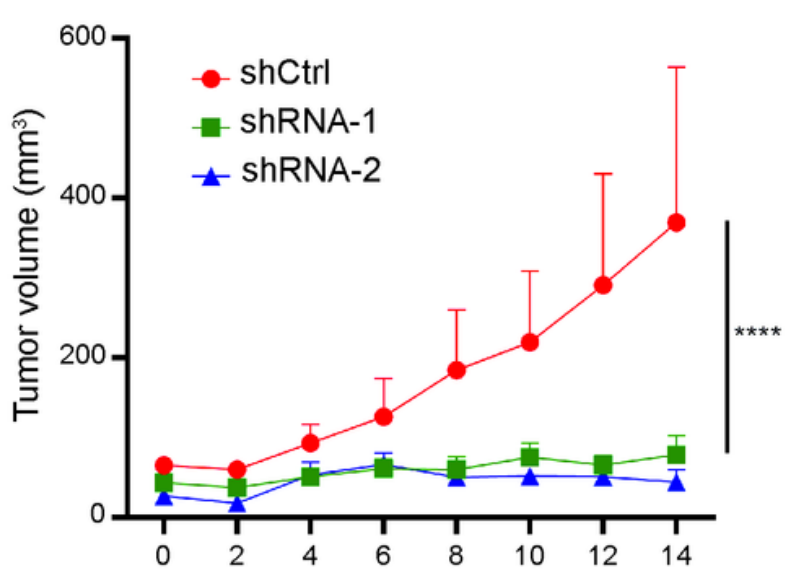

E

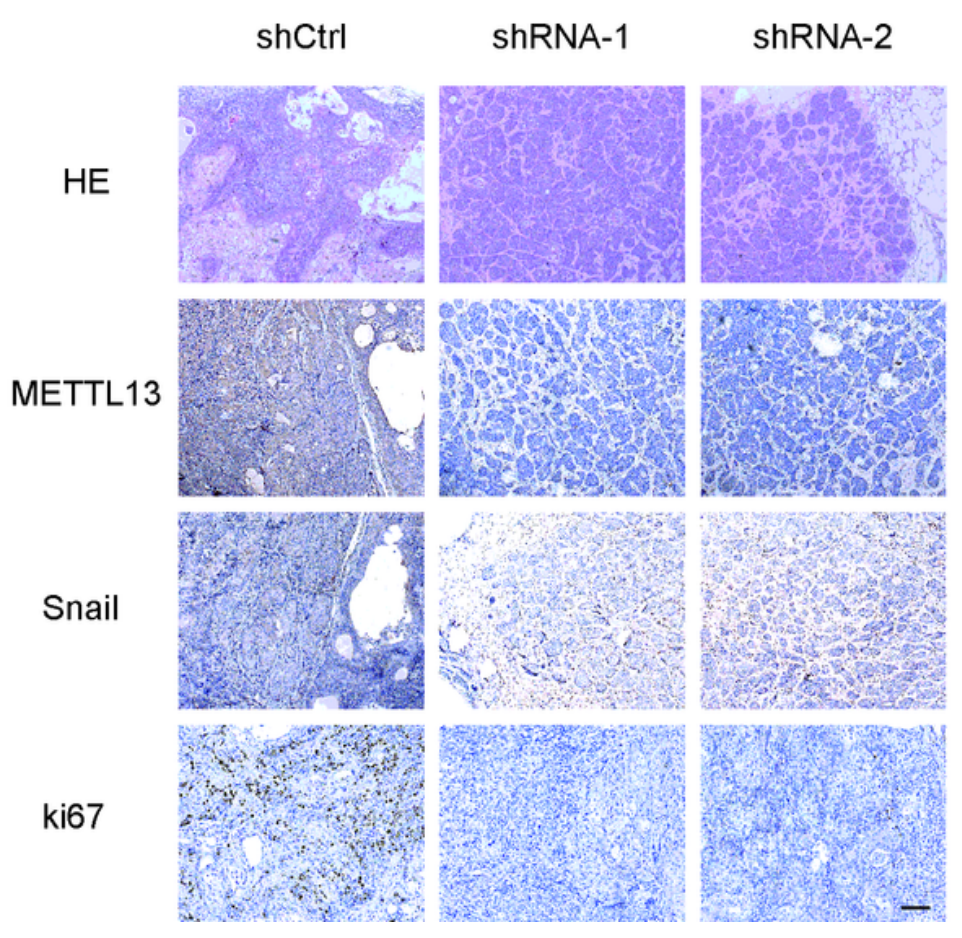


Figure 6

METTL13 regulates HNSCC cell proliferation in vivo. A. BALB/C nude mice $(n=6)$ were subcutaneously transplanted with Cal33 cells transfected with LV-shRNA-1, LV-shRNA-2 and LV-shCtrl. B. Tumors were collected after four weeks. C. Tumor volume was calculated every two days and determined by length $x$ width2/2. D. The tumor weigh was measured after sacrificing mice. E. Representative images of H\&E and immunohistochemistry (IHC) detection of METTL13, Snail and Ki67 in xenograft studies. Scale bar, $200 \mu \mathrm{m}$.

\section{Supplementary Files}

This is a list of supplementary files associated with this preprint. Click to download.

- Table2.pdf

- Table1.pdf 